\title{
Synergistic Place and Object Recognition using Bidirectional Context Information
}

\author{
Sungho Kim and In So Kweon \\ Dept. of EECS, Korea Advanced Institute of Science and Technology \\ 373-1, Guseong-dong Yuseong-gu Daejeon, Korea \\ shkim@rcv.kaist.ac.kr iskweon@kaist.ac.kr
}

\begin{abstract}
In this paper, we present a practical place and object recognition method for guiding visitors in building environments. Due to motion blur or camera noise, places or objects can be ambiguous. The first key contribution of this work is the modeling of bidirectional interaction between places and objects for simultaneous reinforcement. The second key contribution is the unification of visual context, including scene context, object context, and temporal context. The last key contribution is a practical demonstration of the proposed system for visitors in a large scale building environment.
\end{abstract}

\section{Introduction}

Let us imagine that a visitor is looking around a complex building. He might need a guide to get place and object information. This can be realized by using a wearable computer and recent computer vision technology. A web camera on the head receives video data and a wearable computer processes the data to provide place and object recognition results to the person in the form of image and sound in the head mount display (HMD). Visitors with such human computer interaction (HCI) devices can get information on objects and find specific places quickly.

Although the general purpose of place and object categorization is not possible with current state-of-the-art vision technology, recognition or identification of places and objects in certain environments is realizable because of the development of robust local features [6, 7] and strong classifiers like SVM and Adaboost [14]. However, there are several sources of ambiguities caused by motion blur, camera noise, and environmental

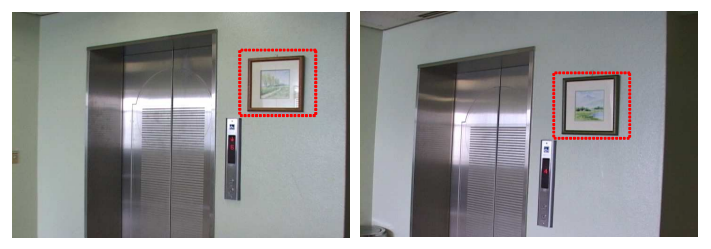

Figure 1: Ambiguity of places by similar environments (Which floor are we at?). This can be disambiguated by recognizing specific objects (pictures on the wall). 


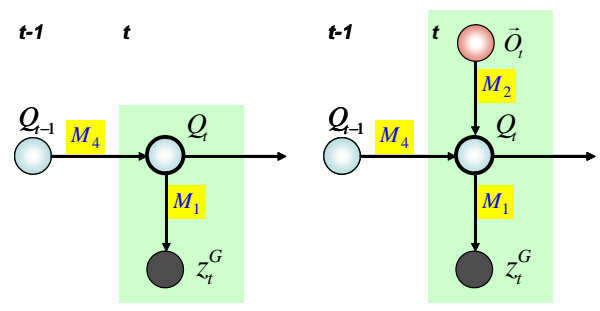

Figure 2: Conventional graphical model (left) and extended graphical model (right) for place recognition in video. Belief at the place node (center circle) gets information from image measurement $\left(M_{1}\right)$, object information $\left(M_{2}\right)$, and previous message $\left(M_{4}\right)$.

similarity. Figure 1 shows an example of place ambiguities caused by similar environments and Figure 5(a) shows an example of ambiguous objects due to blurring. Recently, Torralba et al. and Murphy et al. proposed context-based place and object recognition methods [8, 12]. In [8], Murphy et al. developed a tree structure-based scene, object recognition method by incorporating gist and boosting information. In [12], Torralba et al. utilized gist information from the whole scene. This gist provides strong prior object label and positioning information. These approaches attempted to solve the ambiguity of objects using scene information (Figure 5(a)).

However, no one has tried to disambiguate place label explicitly. Only Torralba incorporated temporal context, which was modeled as the Hidden Markov Model (HMM)[12]. In this paper, we focus on the disambiguation method of simultaneous place and object recognition using bidirectional contextual interaction between places and objects [1]. The human visual system (HVS) can recognize place immediately using a low amount of spatial information. If the place is ambiguous, HVS can discriminate the place using object information in the scene (see the rectangle regions in Figure 1). Motivated from this bidirectional interaction, we present a more robust place and object recognition method.

\section{Place Recognition in Video}

\subsection{Graphical model-based formulation}

Conventionally, place labels from video sequences can be estimated by the well-known Hidden Markov Model (HMM) [12]. We extend the HMM by incorporating the bidirectional context of objects. You can get a clearer concept of the extension through the graphical model, especially Bayesian Net, as shown in Figure 2. A place node at time $t$ is affected by three kinds of information: measurement message (likelihood) from an image, top-down message from objects, and temporal message from the previous state. Let $Q_{t} \in\left\{1,2, \ldots, N_{p}\right\}$ represent place label at $t, z_{t}^{G}$ represent whole image features, $\vec{O}_{t}$ represent object label vector, and $T\left(q^{\prime}, q\right)$ represent the place transition matrix. The Bayesian formula for this graphical model can be represented by equation (1).

$$
\begin{aligned}
& p\left(Q_{t}=q \mid z_{1: t}^{G}, \vec{O}_{1: t}\right) \propto p\left(z_{1: t}^{G} \mid Q_{t}=q\right) p\left(Q_{t}=q \mid \vec{O}_{1: t}\right) p\left(Q_{t}=q \mid z_{1: t-1}^{G}, \vec{O}_{1: t-1}\right) \\
& \text { where } p\left(Q_{t}=q \mid z_{1: t-1}^{G}, \vec{O}_{1: t-1}\right)=\sum_{q^{\prime}} T\left(q^{\prime}, q\right) p\left(Q_{t-1}=q^{\prime} \mid z_{1: t-1}^{G}, \vec{O}_{1: t-1}\right)
\end{aligned}
$$


$p\left(z_{1: t}^{G} \mid Q_{t}=q\right)$ represents bottom-up messages (measurement) from whole images $\left(M_{1}\right), p\left(Q_{t}=q \mid \vec{O}_{t}\right)$ represents top-down messages coming from object label $\left(M_{2}\right), p\left(Q_{t}=\right.$ $\left.q \mid z_{t-1}^{G}, \vec{O}_{t-1}\right)$ represents temporal messages from the previous state $\left(M_{4}\right) . M_{2}$ is calculated by combining messages from related objects using the scene-object compatibility matrix. The important thing from equation (1) is how to utilize individual messages. Combining all the messages is not always a good idea in terms of performance and computational complexity. We can think of three kinds of situations: no temporal context is available (ex. initialization, kidnapped), static context (bottom-up, top-down) is useless due to blurring, and static and temporal context are available and necessary. Since we do not know such situations a priori, we propose a stochastic place estimation scheme as equation (2). $\gamma$ is the probability of reinitialization (mode1) where temporal context is blocked. $\alpha$ is the probability of normal tracking (mode2) where static context is prevented, which reduces the computational load. Otherwise, mode 3 is activated for place estimation. For each frame, each mode is selected according to the selection probability. In this paper, we set the parameters as $\gamma=0.1, \alpha=0.8$ by manual tuning.

$$
\begin{aligned}
& \operatorname{Mode1}(\gamma): p\left(Q_{t}=q \mid z_{1: t}^{G}, \vec{O}_{1: t}\right) \propto M_{1} M_{2} \\
& \operatorname{Mode} 2(\alpha): p\left(Q_{t}=q \mid z_{1: t}^{G}, \vec{O}_{1: t}\right) \propto M_{1} M_{4} \\
& \operatorname{Mode3}(1-\gamma-\alpha): p\left(Q_{t}=q \mid z_{1: t}^{G}, \vec{O}_{1: t}\right) \propto M_{1} M_{2} M_{4}
\end{aligned}
$$

\subsection{Modeling of measurement $\left(M_{1}\right)$}

There are two kinds of place measurement methods depending on feature type. Torralba et al. proposed a very effective place measurement using a set of filter bank responses [12]. In this paper, our place-object recognition system is based on local features [6]. Generalized robust invariant feature (G-RIF) is a generalized version of SIFT [7] by decomposing a scene into convex parts and corner parts, which are described by localized histograms of edge, orientation, and hue. The G-RIF shows upgraded recognition performance by $20 \%$ than SIFT for COIL-100 [6]. For place classification, we utilize the bags of keypoints method proposed by Csurka et al. [2]. Although the SVM-based classification shows better performance than naive Bayes [14], we use a naive Bayes classifier to show the effect of context. The incoming messages from objects or previous places can compensate for the rather weak classifier.

\subsection{Modeling of object message $\left(M_{2}\right)$}

Direct computation of object messages from multiple objects is not easy. If we use the graphical model $[3,15]$, we can estimate approximate messages. Since the number $\left(N_{O}\right)$ of objects and distribution of object nodes are given, the incoming message $M_{2}$ to a place node is expressed as equation (3).

$$
\begin{aligned}
& M_{2}\left(Q_{t}=q\right)=p\left(Q_{t}=q \mid \vec{O}_{t}\right)=\prod_{i=1}^{N_{O}} p\left(Q_{t}=q \mid O_{t}^{i}\right) \\
& \text { where } p\left(Q_{t}=q \mid O_{t}^{i}\right) \propto \max _{k}\left\{\psi\left(q, O_{t}^{i}(k)\right) p\left(O_{t}^{i}(k)\right)\right\}
\end{aligned}
$$




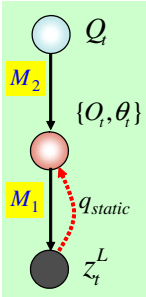

(a)

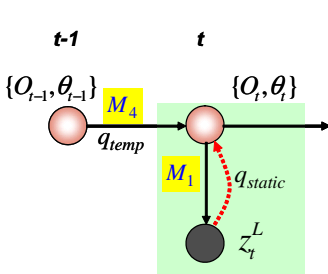

(b)

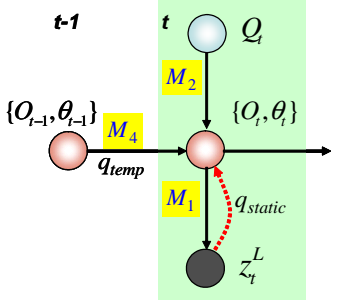

(c)

Figure 3: Graphical model for object recognition in video. In our combined model (c), belief at the object node (center circle) gets information from image measurement $\left(M_{1}\right)$, place information $\left(M_{2}\right)$, and the previous message $\left(M_{4}\right)$.

$O_{t}^{i}(k)$ is a hypothesis of multi-views for $3 \mathrm{D}$ object $O_{t}^{i}$ where $k$ is multi-view index. $p\left(O_{t}^{i}(k)\right)$ is estimated probability of the object hypothesis. $\psi\left(q, O_{t}^{i}(k)\right)$ is the compatibility matrix of the place label and object label. It is estimated by counting the co-occurrences from place-labeled object data. Equation (3) is an approximated version of belief propagation. The max-product method is incorporated instead of the sum-product for the increased estimation accuracy. Physically, the individual maximum message $\left(p\left(Q_{t}=q \mid O_{t}^{i}\right)\right)$ from each object is combined to generate the objects to scene message $\left(M_{2}\right)$.

\subsection{Modeling of temporal message $\left(M_{4}\right)$}

The computation of temporal message from previous place to probable current place is defined as equation (4). It is the same equation used in the HMM [12]. The place transition matrix $T\left(q^{\prime}, q\right)$ is learned by counting frequencies in the physical path. This term prevents quantum jumps during place recognition.

$$
M_{4}\left(Q_{t}=q\right)=p\left(Q_{t}=q \mid z_{t-1}^{G}, \vec{O}_{t-1}\right)=\sum_{q^{\prime}} T\left(q^{\prime}, q\right) p\left(Q_{t-1}=q^{\prime} \mid z_{t-1}^{G}, \vec{O}_{t-1}\right)
$$

\section{Object Recognition in Video}

\subsection{Graphical model-based formulation}

In general, an object node in video can get information from measurement $\left(M_{1}\right)$, message from scene $\left(M_{2}\right)$, and information from the previous state $\left(M_{4}\right)$ as shown in Figure 3 (c), which is combined version of (a) and (b). Murphy et al. proposed a mathematical framework for combining $M_{1}$ and $M_{2}$ with a tree structured graphical model as in Figure 3 (a) [8]. Vermaak et al. proposed a utilization method for combining $M_{1}$ and $M_{4}$ to track multiple objects as Figure 3 (b) [13].

To our knowledge, this is the first attempt to unify these messages within a graphical model. We assume independent objects for simple derivation (see [4] for interaction). This is reasonable since objects are conditioned on a scene. Therefore, we consider only one object for simple mathematical formulation. Let $X_{t}=\left(O_{t}, \theta_{t}\right)$ represent a hybrid state composed of an object label and its pose at $t$. The pose is the similarity transformation 
of an object view. According to the derivation in [11], the complex object probability, given a measurement and place, can be approximated by particles (Monte Carlo) as in equation (5).

$$
p\left(X_{t} \mid z_{1: t}, Q_{1: t}\right) \approx \sum_{i=1}^{N} w_{t}^{i} \delta\left(X_{t}-X_{t}^{i}\right), \text { where } w_{t}^{i} \propto w_{t-1}^{i} \frac{p\left(z_{t} \mid X_{t}^{i}\right) p\left(X_{t}^{i} \mid Q_{t}\right) p\left(X_{t}^{i} \mid X_{t-1}^{i}\right)}{q\left(X_{t}^{i} \mid X_{t-1}^{i}, z_{t}\right)}
$$

As you can see in equation (5), weight is updated by importance sampling where $p\left(z_{t} \mid X_{t}^{i}\right)$ represents measurement $\left(M_{1}\right), p\left(X_{t}^{i} \mid Q_{t}\right)$ represents scene context $\left(M_{2}\right)$, and $p\left(X_{t}^{i} \mid X_{t-1}^{i}\right)$ represents temporal context $\left(M_{4}\right)$ from the previous state. The importance (or proposal) function $\left(q\left(X_{t}^{i} \mid X_{t-1}^{i}, z_{t}\right)\right)$ is defined as equation (6) This is almost the same form as introduced in [10]. The performance of the particle filter depends on modeling the proposal function $(q)$. If we set the proposal function by using only the prior motion, the system cannot cope with the dynamic object appearances and disappearances. Therefore, we utilize the concept of mixture proposal for our problem. If $q_{\text {static }}$ represents the proposal function defined by static object recognition and $q_{\text {temp }}$ represents the proposal function defined by conventional prior motion, then the final proposal is defined as equation (6). Similar to place recognition, we propose three kinds of sample generation modes since we do not know the situation a priori: reinitialization $(r)$ where $\beta=0$, normal tracking $(\alpha)$ where $\beta=1$, and hybrid tracking $(1-r-\alpha)$ where $(0<\beta<1)$. If the temporal context is unavailable, we generate samples from only static object recognition. If in normal tracking mode, we use the conventional proposal function. If static and temporal context are available, we propose samples from hybrid (mixture) density functions.

$$
p\left(X_{t}^{i} \mid X_{t-1}^{i}, z_{t}\right)=(1-\beta) q_{\text {static }}\left(X_{t}^{i} \mid z_{t}\right)+\beta p_{\text {temp }}\left(X_{t}^{i} \mid X_{t-1}^{i}\right)
$$

\subsection{Modeling of proposal function $\left(q\left(X_{t}^{i} \mid X_{t-1}^{i}, z_{t}\right)\right)$}

The proposal function in equation (6) consists of temporal context (prior motion) from the previous state and static context from the input image. The temporal context is modeled as equation (7). We assume that object labels and pose are independent.

$$
p_{\text {temp }}\left(X_{t}^{i} \mid X_{t-1}^{i}\right)=p_{\text {temp }}\left(O_{t}^{(i)} \mid O_{t-1}^{(i)}\right) p_{\text {temp }}\left(\theta_{t}^{(i)} \mid \theta_{t-1}^{(i)}\right)
$$

where $O_{t}^{(i)}=O_{t-1}^{(i)}, t>0$ and $\theta_{t}^{(i)}=\theta_{t-1}^{(i)}+u_{t} . u_{t}$ is Gaussian prior motion. Temporal context means simply normal tracking state.

The static proposal function $q_{\text {static }}\left(X_{t}^{i} \mid z_{t}\right)$ is defined as a Hough transformation using corresponding local features (G-RIF) between image and model with local pose information in the features. We use the scalable 3D object representation and recognition scheme proposed in [5] using shared feature and the view clustering method. Figure 4 graphically summarizes the object recognition procedures. We can get all possible matching pairs by a NN (nearest neighbor) search in the feature library. From these, hypotheses are generated by the Hough transformation in clustered view (CFCM: common frame constellation model) ID, scale (11 bins), orientation ( 8 bins) space, and grouped by object ID. Then, we determine to accept or reject the hypothesized object based on the bin size with an optimal threshold [9]. Finally, we select the optimal hypotheses that can be matched best to the object features in the scene. 


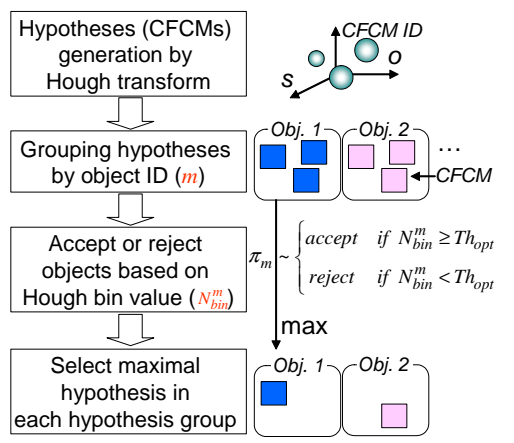

(a)

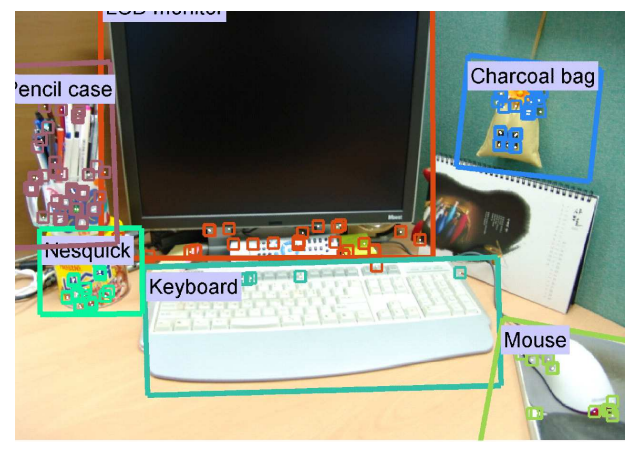

(b)

Figure 4: Hypothesis and test based object recognition procedures (a) and a result (b).

\subsection{Modeling of measurement $M_{1}$}

Given sample object parameters $\left(X_{t}^{i}=\left(O_{t}^{i}, \theta_{t}^{i}\right)\right)$, we use a color histogram in normalized r-g space as a measurement $\left(M_{1}=p\left(z_{t} \mid X_{t}^{i}\right)\right)$. The model color histogram is acquired by the proposal function explained previously since the recognized object can provide an object label with object boundary information. We use $\chi^{2}$ distance in the kernel recipe for measurements [14].

\subsection{Modeling of place message $M_{2}$}

The message from the place to a specific object is calculated using equation (8). We also use approximated belief propagation with the max-product rule.

$$
M_{2}=p\left(O_{t}^{i}, \theta_{t}^{i} \mid Q_{t}\right) \propto \max _{q}\left\{\psi\left(O_{t}^{i}, Q_{t}=q\right) p\left(Q_{t}=q\right)\right\}
$$

\subsection{Modeling of temporal message $M_{4}$}

The temporal message $\left(M_{4}=p\left(X_{t}^{i} \mid X_{t-1}^{i}\right)\right)$ in equation (6) is the same as the proposal function in equation (7). If an object particle is sampled from the temporal context only, the weight is simply the production of measurement and message from place due to the cancellation in equation (6).

\section{Experimental Results}

\subsection{Validation of bidirectional reinforcement property}

First, we test the bidirectional place and object recognition method for ambiguous examples. If we use object information only as in Figure 5(a), we fail to discriminate both objects since the local features are similar. However, if we utilize the bidirectional interaction method, we can discriminate those objects simultaneously as in Figure 5(b).

In the second experiment, we prepare place images taken in front of the elevator at each floor (see example pair in Figure 1). Given a test elevator scene as in Figure 6(a), 


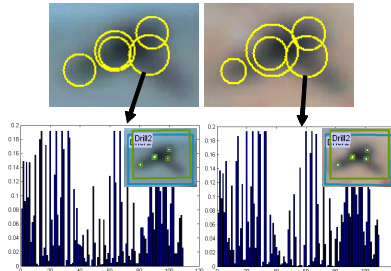

(a)
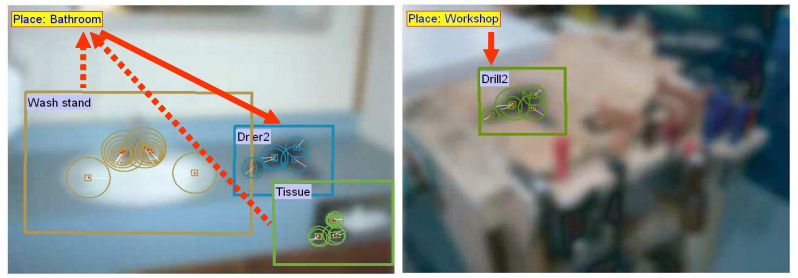

(b)

Figure 5: (a) Object recognition using only object related features (images from [1]) (b) Application of bidirectional interaction between place and objects. Dotted arrows represent objects to place messages to disambiguate place. Solid arrows represent place to object messages to disambiguate blurred objects (dryer, drill).

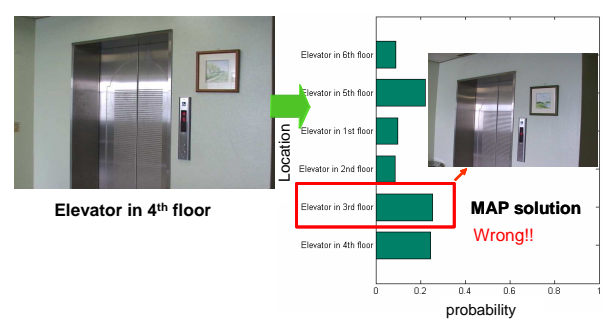

(a)

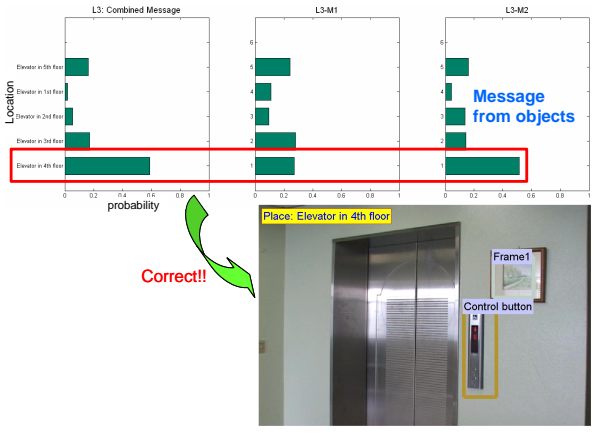

(b)

Figure 6: (a) Place recognition using only measurement $\left(M_{1}\right)$ (b) both measurement and message from objects $\left(M_{2}\right)$

measurement provides recognition of the wrong place. However, if the same test scene is processed using bidirectional place-object recognition, we can obtain correct recognition as in Figure 6(b). In the diagram, the center graph shows the measurement message, the right graph shows the message from the objects, and the left graph represents the combined message for place recognition (see equation (2), Mode 1).

\subsection{Large scale experiment for building guidance}

We validate the proposed place-object recognition system in the department of Electrical Engineering building to guide visitors from the first floor to the third floor. Training data statistics and related images are summarized in Figure 7 and Table 1 respectively. We used 120 images captured at arbitrary poses and manually labeled and segmented 80 objects in 10 places. Note that the images are very blurry. After learning, the size of the object feature was reduced from 42,433 to 30,732 and the scene features were reduced from 106,119 to 62,610 . The number of learned views was reduced from 260 to 209 (2.61 views/object).

In the first experiment, we evaluate the performance of place recognition. As we 

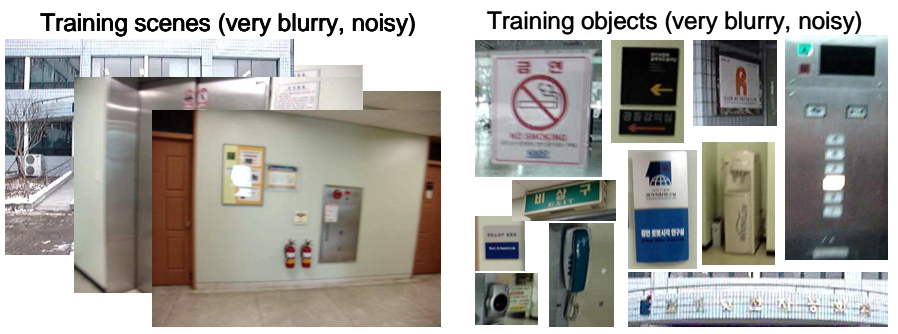

Figure 7: Composition of training and test database.

Table 1: The composition of training images and test images.

\begin{tabular}{|c|c|c|c|c|}
\hline \multirow{2}{*}{ Role } & \multicolumn{2}{|c|}{ Scene $(640 \times 480)$} & \multicolumn{2}{c|}{ Object } \\
\cline { 2 - 5 } & \# of places & \# of scenes & \# of objects & \# of views \\
\hline Training & 10 & 120 & 80 & 209 \\
\hline Test & 10 & 7,208 & 80 & 12,315 \\
\hline
\end{tabular}

said, there are four kinds of message combinations: measurement only $\left(M_{1}\right)$, temporal context (HMM: $\left.M_{1}+M_{4}\right)$, static context only $\left(M_{1}+M_{2}\right)$, and unified context (proposed: $\left.M_{1}+M_{2}+M_{4}\right)$. Figure 8 shows the evaluation results for every 100 frames among 7,208. As you can see, the unified context-based place recognition is better than others.

Figure 9 summarizes the overall evaluation for static context only, full context (static+temporal), and temporal context only. We checked both the detection rate of objects and the relative processing time. The full context-based method shows a better detection rate than the static context only with less processing time. Figure 10 shows partial examples of bidirectional place and object recognition sequences. Note that the proposed system using static context and temporal context shows successful place and object recognition in video under temporal occlusions and with a dynamic number of objects.

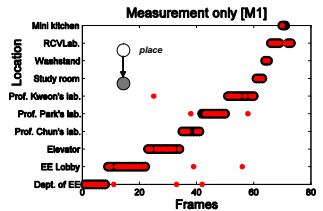

(a)

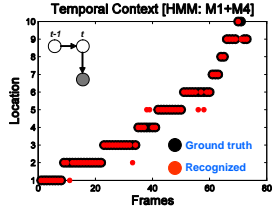

(b)

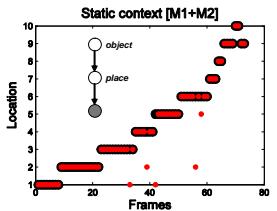

(c)

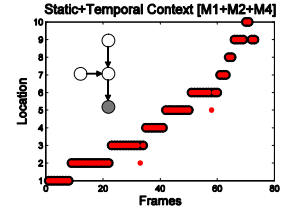

(d)

Figure 8: Place recognition results using (a) measurement, (b) temporal context, (c) static context, and (d) unified context for the test sequences. 


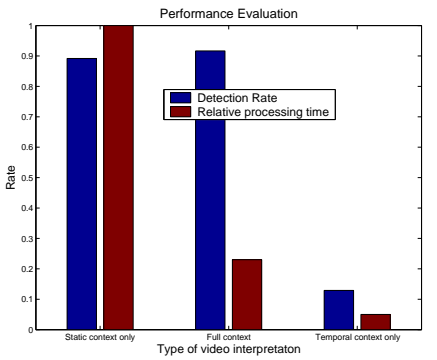

(a)

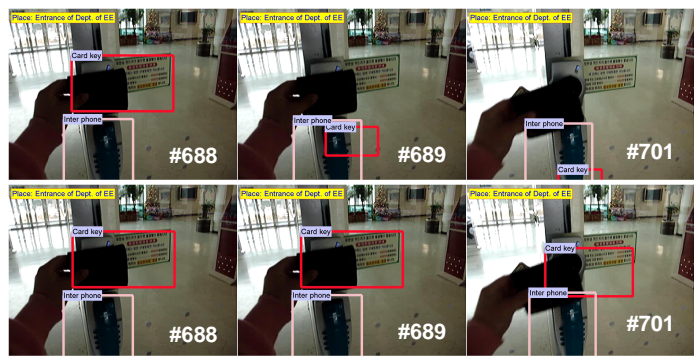

(b)

Figure 9: ( (a) Evaluation of object recognition in terms of detection rate and relative processing time, (b) (Top) Results with only static context, (bottom) using ours.

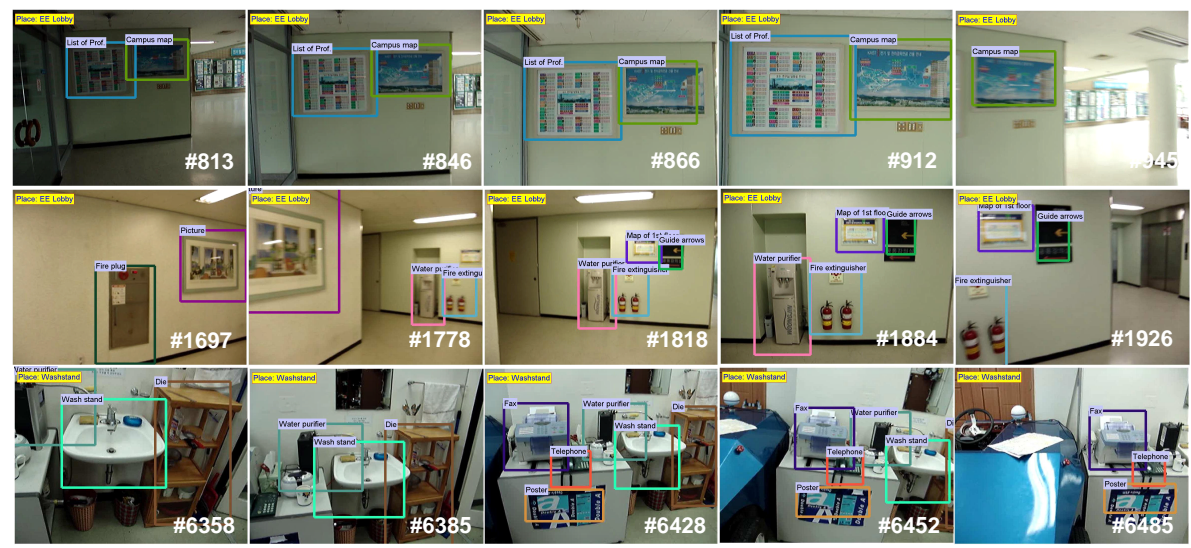

Figure 10: Examples of bidirectional place and object recognition results in lobby and washstand.

\section{Conclusions and Discussion}

In this paper, we present modeling methods of several kinds of visual context focusing on bidirectional interaction between place and object recognition in video. We first modeled object to place messages for the disambiguation of places using object information. The unified context-based place recognition shows improved place recognition. We also modeled place message with measurement and temporal context to recognize objects under the important sampling-based framework. This structure of object recognition in video can disambiguate objects with reduced computational complexity. We demonstrated the synergy of the bidirectional interaction-based place-object recognition system in an indoor environment to guide visitors. The system can be applied directly to various areas for interactions between humans and computers. The proposed method can be upgraded to the category level to provide higher level place and object information to humans. 


\section{Acknowledgements}

This research has been supported by the Korean Ministry of Science and Technology for National Research Laboratory Program (Grant No. M1-0302-00-0064).

\section{References}

[1] M. Bar. Visual objects in context. Nature Reviews: Neuroscience, 5:617-629, 2004.

[2] G. Csurka, C. Dance, C. Bray, and L. Fan. Visual categorization with bags of keypoints. In Workshop on Statistical Learning in Computer Vision, 2004.

[3] M. I. Jordan, editor. Learning in graphical models. MIT Press, Cambridge, MA, USA, 1999.

[4] Z. Khan, T. Balch, and F. Dellaert. MCMC-based particle filtering for tracking a variable number of interacting targets. PAMI, 27(11):1805-1918, 2005.

[5] S. Kim and I. S. Kweon. Scalable representation and learning for 3d object recognition using shared feature-based view clustering. In ACCV (2), pages 561-570, 2006.

[6] S. Kim, K.-J. Yoon, and I. S. Kweon. Object recognition using generalized robust invariant feature and gestalt law of proximity and similarity. In IEEE CVPR Workshop on Perceptual Organization in Computer Vision, 2006.

[7] D. G. Lowe. Distinctive image features from scale-invariant keypoints. Int. J. Comput. Vision, 60(2):91-110, 2004.

[8] K. Murphy, A. Torralba, and W. T. Freeman. Using the forest to see the trees: A graphical model relating features, objects, scenes. In NIPS. 2004.

[9] E. Murphy-Chutorian and J. Triesch. Shared features for scalable appearance-based object recognition. In WACV, pages 16-21, 2005.

[10] K. Okuma, A. Taleghani, N. de Freitas, J. J. Little, and D. G. Lowe. A boosted particle filter: Multitarget detection and tracking. In ECCV (1), pages 28-39, 2004.

[11] N. Gordon Ristic, S. Arulampalam. Beyond the Kalman, Filter-Particle Filters for Tracking Applications. Artech Hous, London, 2004.

[12] A. Torralba, K. P. Murphy, W. T. Freeman, and M. A. Rubin. Context-based vision system for place and object recognition. In ICCV (1), pages 273-280, 2003.

[13] J. Vermaak, A. Doucet, and P. Perez. Maintaining multi-modality through mixture tracking. In $I C C V(2)$, pages 1110-1116, 2003.

[14] C. Wallraven, B. Caputo, and A. Graf. Recognition with local features: the kernel recipe. In $I C C V(1)$, pages 257-264, 2003.

[15] J. S. Yedidia, W. T. Freeman, and Y. Weiss. Understanding belief propagation and its generalizations. Exploring artificial intelligence in the new millennium, pages 239-269, 2003. 